\title{
Capacidades y estrategias de internacionalización del posgrado en la Universidad de Costa Rica
}

Capacities and strategies for postgraduate internationalization

At the University of Costa Rica

\section{Volumen 18, Número 1 \\ Enero-Abril}

pp. 1-33

LATINDEX, DOAJ, REDIB, IRESIE, CLASE, DIALNET, SHERPA/ROMEO, QUALIS-CAPES, MIAR

Revista registrada en los directorios:

ULRICH'S, REDIE, RINACE, OEI, MAESTROTECA, PREAL, $\underline{\text { CLACSO }}$ 


\title{
Capacidades y estrategias de internacionalización del posgrado en la Universidad de Costa Rica

\author{
Capacities and strategies for postgraduate internationalization \\ At the University of Costa Rica
}

\begin{abstract}
Luis Muñoz Varela
Resumen: El presente artículo tiene por finalidad presentar los resultados de una investigación realizada en 2015 y 2016, que tuvo por objetivo hacer un análisis acerca del estado de la cuestión en materia de capacidades y estrategias de internacionalización en los programas de posgrado de la Universidad de Costa Rica (UCR) a escala de la gestión que llevan a cabo las direcciones de los programas. La información recabada se obtuvo por medio de un cuestionario remitido en línea, el cual fue previamente estudiado y validado en 2015 por personal especializado de la Decanatura del Sistema de Estudios de Posgrado (SEP) de la UCR. El análisis efectuado es de carácter descriptivo. Se trata de presentar solo un estado preliminar de la cuestión. Se llevan a cabo, también, observaciones cualitativas en algunos de los aspectos de los resultados de la investigación que más interesaba resaltar. Se concluye con una propuesta de enfoque sobre la internacionalización del posgrado y con indicaciones acerca de las políticas institucionales que en la universidad se podrían formular para mejorar y fortalecer las capacidades y estrategias de gestión, tanto a nivel del SEP como de las direcciones de los diferentes programas de posgrado que integran el Sistema.
\end{abstract}

Palabras clave: internacionalización, movilidad académica, redes académicas, formación profesional de posgrado.

\begin{abstract}
The purpose of this article is to present the results of an investigation carried out in 2015 and 2016 , which aimed to analyze the status of the issue in internationalization capabilities and strategies in the graduate programs of the University of Costa Rica (UCR) at the level of the management carried out by the program departments. The information obtained was obtained through a questionnaire sent online, which was previously studied and validated in 2015 by specialized personnel of the Decentralization of the Graduate Studies System (SEP) of the UCR. The analysis performed is descriptive. It is about presenting only a preliminary status of the issue. Qualitative observations are also carried out on some of the aspects of the research results that were most interesting to highlight. It concludes with a proposal of approach on the internationalization of the postgraduate course and with indications about the institutional policies that in the university could be formulated to improve and strengthen the capacities and management strategies, both at the level of the SEP and the directions of the different graduate programs that make up the System.
\end{abstract}

Key words: internationalization, academic mobility, academic networks, postgraduate professional training.

\footnotetext{
${ }^{1}$ Observatorio de la Educación Nacional y Regional (OBSED), Instituto de Investigación en Educación (INIE), Universidad de Costa Rica (UCR).
}

Dirección electrónica: luis.munoz@ucr.ac.cr

Artículo recibido: 18 de julio, 2017

Enviado a corrección: 9 de octubre, 2017

Aprobado: 18 de diciembre, 2017 


\section{Introducción ${ }^{2}$}

Los procesos de internacionalización de la educación superior han venido desarrollándose en las universidades públicas latinoamericanas desde finales de la década de 1990 (Didou, 2005). La internacionalización hoy se expresa de diversas maneras en la universidad pública, y en torno a ella son observables tendencias diversas y énfasis variados. Es importante indagar cuáles son en cada caso los fundamentos que la internacionalización asume en la universidad pública latinoamericana, sus expresiones y líneas de acción, orientaciones y derivaciones desde el punto de vista de las repercusiones que pueda tener para el quehacer académico y para la gestión que se lleva a cabo en estas instituciones.

En el caso del posgrado, nivel de la formación académica y profesional universitaria que constituye aquí el marco de referencia para el análisis a desarrollar, constituye una dimensión del quehacer universitario especialmente sensible a las tendencias y a los procesos de la internacionalización. En el posgrado:

a) La planta docente tiende a estar integrada por personas que, en su mayoría, han realizado estudios de posgrado en universidades del extranjero; condición que, en principio, es propicia para favorecer las posibilidades de intercambio académico y estudiantil a escala internacional.

b) Existen dinámicas establecidas de movilidad estudiantil hacia el exterior para participar en estancias cortas o pasantías, incluidas como componentes o requisitos de los planes de estudio.

c) El posgrado es una dimensión del quehacer universitario en la que existe una tendencia mayor a participar en redes académicas internacionales (formales e informales), en docencia, investigación, acción social, gestión académica y otras.

d) Las dinámicas de intercambio académico son, por lo general, más intensas en el posgrado que en el grado o pregrado.

En virtud de lo anterior, es importante en la Universidad estudiar las capacidades y las estrategias de internacionalización con que se cuenta, a escala de la gestión del posgrado. Esto permite identificar cuál es la dimensión y la naturaleza de dichas capacidades y

2 La presente investigación se realizó en el marco de la participación que el Instituto de Investigación en Educación (INIE) de la Universidad de Costa Rica, mantiene en la Red sobre Internacionalización y Movilidades Académicas y Científicas (RIMAC) del Centro de Investigación y Estudios Avanzados (CINVESTAV), apoyada por el Programa de Redes Temáticas del Consejo Nacional de Ciencia y Tecnología, México 
estrategias, sus orientaciones, relaciones internacionales establecidas, alcances que tiene para la mejora de la excelencia y la pertinencia social e institucional del quehacer académico.

La UCR cuenta con 6 áreas académicas y un área de Sedes Regionales. Las áreas académicas son: Ciencias Agroalimentarias, Ciencias Básicas, Artes y Letras, Ingeniería y Arquitectura, Ciencias de la Salud y Ciencias Sociales. De conformidad con información obtenida de la Decanatura del Sistema de Estudios de Posgrado (SEP) de la UCR, al año 2017 existen, en la Universidad, 70 programas de posgrado, distribuidos por áreas académicas según se muestra en la Figura 1

Figura 1

Número de programas de posgrado de la UCR por área académica. Periodo 2015-2016.

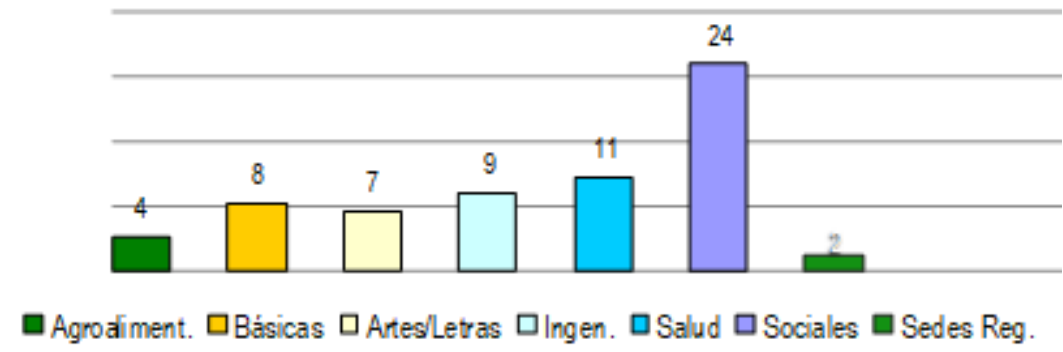

Fuente: Elaboración propia con base en información disponible en la página web del SEP.

La investigación, realizada en 2015 y 2016, cuyos resultados se presentan aquí, partió de una base de 66 programas de posgrado, la cual era la cantidad existente a ese momento. Los resultados expuestos a continuación se basan, en consecuencia, en esa cantidad de 66 programas. Asimismo, es importante indicar que entre los 70 programas de posgrado actualmente existentes en la universidad, 7 de ellos están clasificados como "programas interdisciplinarios" y, por lo tanto, no aparecen registrados en la Figura 1.

\subsection{Distribución de programas de posgrado por facultades}

Las áreas de conocimiento están integradas por una o más facultades; 3 de ellas están conformadas por una sola facultad: Ciencias Agroalimentarias, Ingeniería y Arquitectura y Ciencias Básicas. Por su parte, de las otras 3, Bellas Artes está conformada por 2 facultades (Facultad de Artes y Facultad de Letras); Ciencias de la Salud cuenta con 4: Facultad de Medicina, Facultad de Odontología, Facultad de Microbiología y Facultad de Farmacia. 
Finalmente, el área de Ciencias Sociales está conformada también por 4 facultades: Facultad de Educación, Facultad de Derecho, Facultad de Ciencias Económicas y Facultad de Ciencias Sociales.

La distribución por facultades de los 66 programas de posgrado presenta una relación algo más equilibrada que la distribución por áreas académicas, según la dimensión o cantidad de unidades académicas que conforman cada una de ellas. En la Figura 2 se presentan las correspondientes distribuciones.

Figura 2

Número de programas de posgrado de la UCR por facultad. Periodo 20152016.

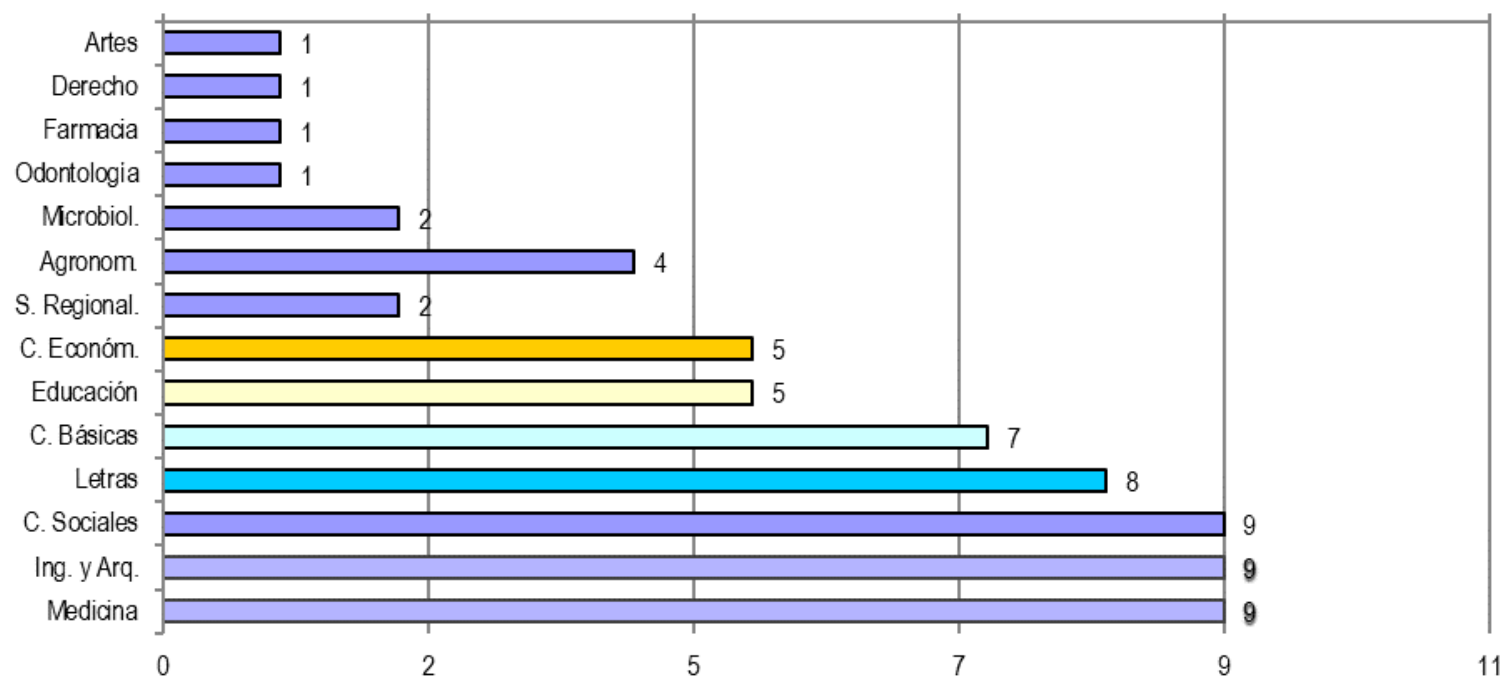

Fuente: elaboración propia con información proporcionada por la Decanatura del SEP.

Cabe precisar que en los programas de posgrado pueden estar incluidas una o más titulaciones distintas. Por ejemplo: un programa como el Posgrado en Administración Universitaria cuenta con una única titulación (maestría profesional); otro programa, el Posgrado en Derecho, cuenta en su lugar con 13 titulaciones: 1 doctorado, 9 maestrías y 3 especialidades.

El presente artículo expone los resultados de una investigación llevada a cabo en 2015 y 2016, en relación con diversos aspectos de la internacionalización del posgrado en la UCR: formación de posgrado en universidades del extranjero, movilidad docente y estudiantil entrante y saliente, intercambio académico, participación en redes internacionales, convenios que se tienen suscritos con universidades del extranjero y otros. El análisis de los resultados 
es de índole básicamente descriptiva, en la medida en que se trata de una aproximación al estado de la cuestión.

\section{Referencialidad teórica}

\subsection{Planteamiento general}

En América Latina, algunos países con mayor énfasis que otros, desde la década de 1990 son escenario de una diversa preocupación por el análisis y la discusión acerca de la naturaleza, visión, prácticas, políticas y cursos de acción de la internacionalización de la educación superior (Albizu, 2014). Esto ocurre como parte de lo que Lanz, Fergusson y Marcuzzi (2006) denominan el "tercer proceso de reforma universitaria" en América Latina, caracterizado por "enormes transformaciones sociales, políticas, económicas y culturales que dieron paso a los nuevos tiempos, la globalización, la mundialización y la internacionalización de la educación superior [...]" (p. 108).

En términos generales:

La tecnología de la información, la economía del conocimiento, la movilidad cada vez mayor de los estudiantes, profesores, programas y proveedores y la integración creciente de la economía mundial son factores que estimulan esta internacionalización. Sin duda, la tendencia a la internacionalización seguirá siendo una fuerza central en la educación superior para el futuro inmediato. (Altbach y Knight, 2006, p. 36)

Hasta ahora, la tendencia de mayor predominancia en internacionalización seguramente sea la de la transnacionalización mercantil de los servicios de educación superior, tendencia asociada intrínsecamente a las políticas de la Organización Mundial del Comercio (OMC), dirigidas a transformar la educación superior - y la educación en general - de ser un bien público brindado por el Estado a un servicio privado con naturaleza de mercado. Distintos organismos multilaterales han diseñado estrategias, programas y mecanismos para impulsar e instituir, en la educación superior, la internacionalización mercantil: Organización para la Cooperación y el Desarrollo Económico (OCDE), la Unión Europea, el Proceso de Bolonia (Dias, 2008). En América Latina, esta tendencia de internacionalización ha generado preocupación y reacciones de diversa índole, tanto teóricas como institucionales, sobre todo cuando se trata de tendencias que de una u otra forma afectan, en su cumplimiento, los principios, fines y misiones de las universidades públicas. 
Los detonantes de la internacionalización mercantil se basan en las reglas de disposición, generación, control y distribución de la "tecnociencia hegemónica" (Vessuri, 2003), generada en los países hegemónicos y rectores de la actual economía global de capitalismo corporativo.

La idea de las universidades como grandes proyectos sociales 0 instituciones culturales para la producción de bienes públicos se ha convertido en un campo marginal o meramente discursivo y ha sido reemplazada por un renovado énfasis en los vínculos entre la educación superior y los mercados. (Escrigas y Lobera, 2009, p. 9)

Se trata de una organización epistémica y programática del saber cuyas codificaciones se despliegan a escala global planetaria, instituyendo referencialidades y demandas de formación profesional estandarizada, traducidas en sistemas internacionales de evaluación y acreditación de la educación superior. Tienden a imponer determinadas agendas, políticas y cursos de acción para la investigación universitaria, que priorizan la investigación aplicada y con preferencia asociada a la empresa privada industrial vinculada al mercado global. Institucionalizan parámetros de medición de la calidad con base en indicadores de evaluación y acreditación internacionales definidos en las oficinas "especializadas" de los organismos multilaterales promotores de la globalización económica y de la competitividad como principio que debe regir también para la prestación de los servicios de educación superior. Establecen normas y controles exógenos sobre las publicaciones nacionales por medio de determinados requisitos para elaborar un artículo que pueda ser calificado como publicación "científica", entre otros.

En la medida en que la universidad pública adopta tales orientaciones para definir y direccionar su quehacer académico (formación profesional, investigación, acción social), tanto a nivel de grado como de posgrado se abre paso una desterritorialización de los principios y finalidades que dan fundamento a una labor institucional orientada a atender las necesidades diversas que la sociedad afronta, en la perspectiva de "contribuir con las transformaciones que la sociedad necesita para el logro del bien común" (Consejo Universitario de la Universidad de Costa Rica, 2005, p. 1).

Según señala Mollis (2010b), en América Latina, las políticas públicas sobre educación superior de los últimos años: 
[...] impactaron el sentido y la misión de las universidades, volviéndolas cada vez más instituciones terciarias para el entrenamiento profesional y menos universidades en el sentido del saber para transformar, hacer ciencia y socializar a favor de una identidad ciudadana crítica. (p. 12)

La conformación de contenido y finalidad de la formación profesional y la heurística de la investigación tienden a retraer su responsabilidad con respecto a atender las realidades nacionales de la pobreza; la complejidad de necesidades de saber y tecnológicas que existen en la matriz productiva de cada país; la inequidad y la exclusión social y productiva estructural; la discriminación étnica y cultural; la educación estandarizada y la privatización de los servicios educativos; el énfasis en el esquema de la productividad económica local basada en el neoextractivismo, con sus repercusiones destructivas para el medio ambiente y los recursos naturales; la agroindustria de monocultivo transnacional para la exportación; la pérdida de soberanía alimentaria y su reemplazo por la dependencia externa para el abastecimiento; la corporativización segmentada de la producción nacional y el orillamiento de las pequeñas y medianas unidades productivas. En fin, problemas todos estos que, de manera crítica, tendrían que constituir componentes esenciales en la definición y conformación de los planes de estudio de la formación académica y profesional del posgrado, de igual manera que en la investigación y en la acción social.

A partir de la década de 1990, la oferta de estudios de posgrado en las universidades de América Latina se expandió de manera muy significativa (Mollis, 2010a), asociada, en general, a las políticas que los gobiernos y distintos organismos multilaterales promovieron en el sentido de vertebrar la formación profesional de posgrado con las demandas del mercado de trabajo de la economía transnacionalizada y global, fundamentada esta de manera intensiva en las innovaciones de la revolución científico/tecnológica.

En consecuencia, como tendencia que marca a las universidades de América Latina durante los últimos años, se observa la prioridad de dar apertura a posgrados "orientados a cubrir áreas de actualización profesional en desmedro de los posgrados académicos de carácter científico" (Mollis, 2010a, p. 15). Con ello, la pertinencia social del posgrado se diluye y pasa a convertirse en una formación para una élite profesional que ya no se reconoce, sino inscrita en la lógica de conseguir un empleo en la gran empresa nacional industrial, o bien en alguna corporación transnacional de inversión extranjera directa. Puede agregarse, además, que esta situación también favorece condiciones para que en los países 
se incremente la así denominada "fuga de cerebros", que ahora ya no solo involucra el traslado al extranjero, sino que también se produce al interior mismo de cada país.

En ese contexto, el posgrado se desplaza a adoptar e incorporar determinadas demandas externas para la formación profesional, predominantemente inscritas en la lógica de la competitividad interinstitucional que se intensifica conforme se amplía y consolida el mercado de las titulaciones profesionales de posgrado. De manera concomitante, esta situación tiende a la vez a reconfigurar, en el posgrado, las agendas y las dinámicas de la investigación, así como de la oferta académica y la investigación universitaria en general. Las políticas institucionales de investigación pasan, incluso, a ser redefinidas sobre la marcha, de facto, sin mediar análisis ni consulta con las correspondientes comunidades universitarias. Se filtra, localmente y sin mayor reparo, el imaginario de que el camino ahora está trazado por la mercantilización del conocimiento y por el desarrollo del ethos de la competitividad, la innovación y el emprendedurismo.

La política institucional pasa, entonces, a ser definida de manera vertical por las altas autoridades universitarias, bajo el signo de las indicaciones que irradian externamente desde los centros hegemónicos del poder científico/tecnológico y del mercado globalizado, que de esa forma buscan instituir su propia racionalidad y control sobre la producción académica y científica local de las naciones.

En una posición distinta, contrapuesta a la predominante internacionalización de carácter mercantil, en la universidad latinoamericana también emergen voces que abogan por una perspectiva de relaciones interinstitucionales basada en la cooperación internacional solidaria y colaborativa, horizontal y de beneficio recíproco, donde se tenga en cuenta la importancia de fortalecer las relaciones, intercambios y acciones conjuntas a escala regional y también en sentido Sur/Sur. Desde esta perspectiva, la internacionalización pasa a ser valorada como importante en la medida que puede contribuir al fortalecimiento de los intercambios interinstitucionales a nivel regional, en búsqueda de afrontar y generar alternativas conjuntas a la tendencia de internacionalización mercantilizante y corporativa.

\subsection{Políticas de internacionalización en la UCR}

En el caso de la UCR, el Plan Estratégico Institucional (PEI) 2013/2017 coloca a la internacionalización como el cuarto de seis ejes estratégicos, con respecto al cual se plantea como principal finalidad la siguiente: 
Establecer redes de cooperación e intercambio que consoliden la posición de la UCR en el escenario académico internacional y fomenten la movilidad activa de docentes, estudiantes y personal administrativo, enriqueciendo nuestro acervo científico y cultural, a partir del contacto con la diversidad de experiencias. (Oficina de Planificación Universitaria, s. f., p. 18)

Se estipulan también los siguientes dos objetivos específicos:

- Fomentar el desarrollo de redes académicas -formales y no formales-, en los ámbitos nacional e internacional.

- Fomentar la movilidad de docentes, estudiantes y personal administrativo tanto nacional como internacionalmente. (Oficina de Planificación Universitaria, s. f., p. 18)

Las acciones indicadas en el PEI 2013-2017, para el cumplimiento de estos objetivos, colocan sus énfasis en: a) participación en redes internacionales de investigación, formales y no formales; b) participación del $20 \%$ de la comunidad investigadora de la UCR en proyectos innovadores con redes internacionales; c) incremento de los convenios con macrouniversidades de prestigio internacional; d) movilidad docente, estudiantil y administrativa; e) académicos visitantes (Oficina de Planificación Universitaria, s. f.).

A propósito del tema de las redes académicas internacionales, en 2009, la Declaración Final de la Conferencia Regional de la Educación Superior (CRES) en América Latina y el Caribe (Cartagena de Indias, Colombia) señaló lo siguiente:

Es mediante la constitución de redes que las instituciones de Educación Superior de la región pueden unir y compartir el potencial científico y cultural que poseen para el análisis y propuesta de solución a problemas estratégicos. Dichos problemas no reconocen fronteras y su solución depende de la realización de esfuerzos mancomunados entre las instituciones de Educación Superior y los Estados. (CRES, 2008, p. 8)

Según se puede notar, la CRES (2008) coloca el acento en el contexto de la regionalidad como el punto de partida más apropiado para el desarrollo de acciones interinstitucionales en red por parte de las universidades de América Latina y el Caribe.

Las redes académicas a escala nacional y regional son interlocutores estratégicos ante los gobiernos. Son, asimismo, los protagonistas indicados para articular de manera 
significativa identidades locales y regionales, y colaborando activamente en la superación de las fuertes asimetrías que prevalecen en la región y en el mundo frente al fenómeno global de la internacionalización de la Educación Superior. (p. 8)

A fin de dar concreción a estos enunciados, la Declaración de la CRES define un marco de acción conjunta a escala regional al que denomina Espacio de Encuentro Latinoamericano y Caribeño de la Educación Superior (ENLACES):

Es fundamental la construcción de un Espacio de Encuentro Latinoamericano y Caribeño de Educación Superior (ENLACES), el cual debe formar parte de la agenda de los gobiernos y los organismos multilaterales de carácter regional. Ello es básico para alcanzar niveles superiores que apunten a aspectos fundamentales de la integración regional: la profundización de su dimensión cultural; el desarrollo de fortalezas académicas que consoliden las perspectivas regionales ante los más acuciantes problemas mundiales; el aprovechamiento de los recursos humanos para crear sinergias en escala regional; la superación de brechas en la disponibilidad de conocimientos y capacidades profesionales y técnicas; la consideración del saber desde el prisma del bienestar colectivo; y la creación de competencias para la conexión orgánica entre el conocimiento académico, el mundo de la producción, el trabajo y la vida social, con actitud humanista y responsabilidad intelectual. (p. 9)

Al respecto, la CRES puntualiza varias acciones que las universidades tendrían que asumir y desarrollar:

e. el fomento de la movilidad intrarregional de estudiantes, investigadores, profesores y personal administrativo, incluso, a través de la implementación de fondos específicos;

f. el emprendimiento de proyectos conjuntos de investigación y la creación de redes de investigación y docencia multiuniversitarias y pluridisciplinarias;

g. el establecimiento de instrumentos de comunicación para favorecer la circulación de la información y el aprendizaje;

i. el fortalecimiento del aprendizaje de lenguas de la región para favorecer una integración regional que incorpore como riqueza la diversidad cultural y el plurilingüismo. (CRES, 2008, pp. 23-24) 
En contexto de internacionalización de la educación superior, las redes pueden ser aprovechadas en el posgrado para enriquecer el quehacer académico y la formación de alto nivel, se hace énfasis en posicionar los contenidos que releven la preocupación por estudiar y colocarse en los propios contextos de la realidad nacional incorporando al mismo tiempo los escenarios de lo regional, lo internacional y lo global.

[...] la mayoría de las disciplinas y el grueso de los tipos de producción del conocimiento se basan crecientemente en redes internacionales, y la tendencia entre los académicos es a identificarse cada vez más con comunidades, redes e instituciones internacionales que lo que se acostumbraba en el pasado. (Vessuri, 2008, p. 128)

Los grandes problemas que hoy afectan a la humanidad son comunes a escala planetaria, pero tienen expresiones diversas según las regiones. La pobreza, el hambre, la guerra, el racismo, los fundamentalismos, el consumismo, la explotación sin control de los recursos naturales y la destrucción del medio ambiente, las migraciones, la corrupción institucionalizada en lo público y lo privado, el incremento de las desigualdades sociales estructurales..., aunque comunes a escala planetaria, las formas de afrontar y buscar respuestas a estos problemas globales no dejan de estar situadas en los propios contextos nacionales de cultura, saber y hacer de cada país, lo cual no debe obviarse al actuar de manera mancomunada a nivel internacional y regional.

Desde las propuestas alternativas a la internacionalización mercantil, la finalidad fundamental es contribuir a atender y buscar soluciones a los problemas locales de manera mancomunada y convergente, para afrontarlos y atenderlos cada universidad y cada sistema universitario nacional, a partir de su propia contextualidad e identidades institucionales y culturales; buscando relevar, enriquecer y validar las propias capacidades y acervos de saber con que se cuenta. La validación y consolidación de las capacidades diversas de saber que cada sociedad y cada colectivo humano, étnico y cultural posee, se ve restringida y obstaculizada cuando el saber se instaura como un saber externo, hegemónico, inscrito en una lógica de estandarización utilitaria y mercantil.

A propósito de otra de las cuestiones incluidas en el PEI 2013/2017 de la UCR, la movilidad académica docente, estudiantil y administrativa, las iniciativas y acciones de internacionalización pueden contribuir a amplificar de manera apropiada el registro de las relaciones con diversas universidades y otras instituciones del exterior. Esto permite identificar opciones alternativas que contribuyan a fortalecer las posibilidades de intercambio 
y de trabajo interinstitucional conjunto y colaborativo. Los intercambios contribuyen, además, a ampliar la visión de mundo, a contar con elementos para valorar la propia cultura local y nacional, lo cual favorece, también, que las personas puedan conocer otras formas de acceder al análisis de los problemas en los ámbitos disciplinares a los que se dedican y en los que se están formando.

En ese sentido, las finalidades de la movilidad deberían ser organizadas siguiendo criterios de óptimo aprovechamiento de los recursos y las oportunidades existentes. Por ejemplo, una cuestión central sería la de conjugar los intereses individuales de las personas, en relación con las de la formación de alto nivel que el país amerita para fortalecer y potenciar las propias capacidades endógenas que se requiere incrementar y consolidar en las diferentes áreas del saber: sociales, humanísticas, culturales, científicas y tecnológicas.

Naturalmente, no se trataría tampoco de agendar e imponer reglas rígidas que a la postre terminarían resultando excluyentes o incluso violatorias de los derechos individuales de las personas. Lo que parece razonable y apropiadamente universitario es, en cambio, asegurar la convergencia de los distintos intereses. Evitar que los intereses se encaucen por una tangente de beneficios de alcance unilateral, esa es la cuestión que requiere ser dilucidada. $\mathrm{Y}$ esto se puede lograr por medio de políticas institucionales de movilidad basadas en objetivos claros y transparentes, de la misma manera que en la flexibilidad de gestión necesaria para garantizar una dinámica de diálogo entre las partes involucradas y para la toma razonada, ponderada y satisfactoria de las decisiones. De lo que se trata es de desarrollar una movilidad enmarcada en la conjugación de la doble dimensión del interés personal individual y de intereses y políticas institucionales acordes y congruentes con la misión institucional de aportar a la sociedad los recursos y los conocimientos que esta necesita para el fortalecimiento de la institucionalidad democrática y para contribuir a la generación de las condiciones que permitan el desarrollo de las capacidades de diversa índole requeridas para garantizar el bienestar y una vida digna para toda la sociedad en general (Nussbaum, 2012).

Las acciones de internacionalización requieren hoy ser organizadas en políticas institucionales integradas. La iniciativa individual resulta siempre valiosa, pero no puede dejarse a su propia suerte sin que se le proporcione el apoyo institucional adecuado. Es importante, por consiguiente, que en la universidad exista una política institucional definida de internacionalización, cuya formulación y establecimiento sea, además, producto de un amplio y diverso proceso de consulta con las diferentes unidades académicas y los 
diferentes sectores que conforman la comunidad universitaria.

Por su propia naturaleza, el posgrado está llamado a constituir una de las dimensiones del quehacer académico universitario en la que de manera preferente se desarrollen iniciativas y acciones de internacionalización. Esto es especialmente importante en lo que corresponde al aspecto de la investigación, pero también lo es por medio del intercambio académico internacional en materia de docencia. De igual manera, también cabe mencionar los convenios que las carreras puedan tener suscritos con centros de investigación y unidades académicas de universidades del extranjero.

Las acciones de internacionalización pueden posibilitar a las carreras de posgrado disponer de espacios para desarrollar iniciativas de intercambio en materia de investigación; experiencias y nuevos enfoques curriculares; pertinencia y relevancia de los estudios de posgrado para un desarrollo nacional equitativo, con justicia social, democrático, solidario, incluyente e integral; contribución de las personas tituladas de posgrado en los ámbitos institucionales, económicos, productivos, científicos y de intervención en diferentes campos de actividad; movilidad estudiantil y académica; capacitación para el fortalecimiento de la enseñanza y de los perfiles de la planta académica en general.

Para tal efecto y, además, teniendo en cuenta que la internacionalización ha pasado a constituir uno de los ejes incluidos en el Plan Estratégico Institucional 2013/2017 de la UCR, la finalidad de la presente investigación ha sido la de conocer cuál es la situación actual, que presentan las carreras de posgrado de la UCR, en lo que concierne al desarrollo de capacidades y estrategias para la internacionalización del currículum, la investigación, la participación en redes académicas internacionales y la formación de su planta académica y la de sus estudiantes.

\section{Metodología}

La investigación se llevó a cabo por medio de:

a) Investigación bibliográfica acerca de experiencias de internacionalización de la educación superior desarrolladas en América Latina, aunque también se revisó bibliografía, sobre esta temática, publicada por investigadoras e investigadores de América del Norte y Europa. La información obtenida sirvió para elaborar el marco teórico y para definir la perspectiva de internacionalización a asumir como referencialidad teórica. 
b) Se elaboró la propuesta del proyecto de investigación y se diseñó el respectivo instrumento, consistente en un cuestionario a remitir en línea por medio del programa "LimeSurvey".

c) Se contactó con la Decanatura del Sistema de Estudios de Posgrado (SEP) para solicitar una reunión y exponer la propuesta del proyecto. Esta reunión se llevó a cabo y sirvió para obtener información acerca de la labor que se desarrolla en la Decanatura, así como sobre algunas características generales de los programas de posgrado. También se logró obtener la colaboración de una de las funcionarias de la Decanatura para participar en la revisión y validación del instrumento de investigación.

d) Una vez aplicado el instrumento de investigación y registradas las respuestas obtenidas a lo largo de 2 meses, se procedió a elaborar con ellas un registro en formato "Excel".

e) Se procedió a la sistematización de la información recibida y a la correspondiente elaboración integrada de los resultados.

\section{Resultados}

Del total de los 66 programas de posgrado de la UCR, se obtuvo respuesta al cuestionario por parte de las direcciones de 38 de ellos (55\%). Los programas del área de Ciencias Sociales son los que proporcionalmente presentaron una mayor disposición para responder al cuestionario, mientras que en el área de Ciencias Básicas fue donde se registró la menor proporción de respuestas obtenidas.

\subsection{Experiencia de estudios en el extranjero y de movilidad e intercambio académico internacional}

\subsubsection{Universidad donde obtuvo el último grado de titulación}

Del conjunto de las 38 personas directoras de programas de posgrado que respondieron al cuestionario, 26 de ellas (68,42\%) indican haber obtenido su último grado de titulación en una universidad del extranjero. En total, son 24 universidades extranjeras, distribuidas en una pequeña cantidad de 8 países. Únicamente 2 de esas universidades se repiten (una sola vez) en la información obtenida: Universidad Autónoma de Barcelona (España) y Universidad de Montreal (Canadá).

La distribución por países de las 24 universidades extranjeras es la siguiente (figura 3): 
Figura 3

Número de directoras y directores del programa de posgrado de la UCR por países de destino en donde obtuvieron su última titulación en una universidad extranjera. Periodo 2015-2016.

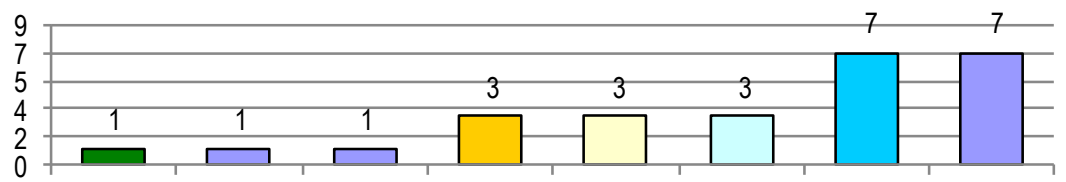

Suiza Países BajosColombia Canadæstados Unido§Francia Alemania España

Fuente: elaboración propia con base en la información obtenida de parte de las Direcciones de Posgrado informantes.

Una única universidad de América Latina figura en este conjunto de universidades extranjeras, la Universidad del Valle (Cali). En su lugar, hay una alta concentración (73,07\%) en universidades de Europa, mientras que América del Norte (con participación especialmente de universidades de Estados Unidos y Canadá) registra una proporción de $23,07 \%$.

Según lo anterior, puede decirse que en la UCR prevalecen las rutas tradicionales que en América Latina son características cuando se trata de elegir destinos para realizar estudios de posgrado en el exterior. La información obtenida también se ve significativamente reflejada en la que, por su parte, proporciona la Oficina de Asuntos Internacionales y Cooperación Externa (OAICE) de la UCR, en relación con la cantidad total de docentes que en el año 2015 salieron a realizar estudios de posgrado en el exterior con apoyo complementario de una beca de la institución. La cifra total en este registro de la OAICE es de 52 personas, cuyas universidades de destino se distribuyen por regiones geográficas de la siguiente manera: a) Europa: 64\%; b) América del Norte: 19\%; c) América Latina: 17\%. (Carranza, 2015, p. 21). Los países de destino que ocupan las primeras posiciones en este registro son, respectivamente: Estados Unidos, Alemania, España, Inglaterra y Francia. Esta situación se ve, en este momento, alentada por la política y el PEI 2013/2017 de la UCR, que en su eje cuarto (Internacionalización) estipula dar prioridad al establecimiento de convenios con "macrouniversidades de prestigio internacional" (Oficina de Planificación Universitaria, s. f., p. 18).

\subsection{Relaciones con colegas de la universidad extranjera donde se realizaron los estudios del último grado de titulación}

Respecto de las relaciones que estas personas mantienen con colegas de la universidad extranjera donde obtuvieron su última titulación, el 53,84\% menciona que 
mantienen relaciones constantes o frecuentes. Del restante $46,15 \%$ de las personas, $26,92 \%$ indica que solo mantiene relaciones esporádicas y un $19,23 \%$ señala que no mantiene ningún tipo de relación. Si se considera que esta información incluye una proporción de 80\% de las personas que refieren mantener relaciones constantes, periódicas, o bien, esporádicas con colegas de la universidad extranjera donde obtuvieron su última titulación, puede entonces decirse que en las direcciones de los programas de posgrado de la UCR existe un potencial importante de experiencia de relaciones internacionales que puede ser aprovechado para fortalecer los intercambios internacionales en los programas de posgrado.

\subsection{Actividades que se llevan a cabo en el marco de la relación establecida con colegas de la universidad extranjera donde se obtuvo el último grado de titulación}

Según la información obtenida, son diversas las acciones y actividades que las personas informantes indican llevarse a cabo en el marco de las relaciones establecidas con colegas de la universidad donde realizaron sus estudios en el extranjero (figura 4). Entre ellas, destacan el intercambio docente, la realización de conferencias y seminarios, así como las publicaciones conjuntas.

Figura 4

Actividades que las personas directoras de posgrado de la UCR llevan a cabo en conjunto con colegas de la universidad extranjera donde obtuvieron el último grado de titulación. Periodo 20152016.

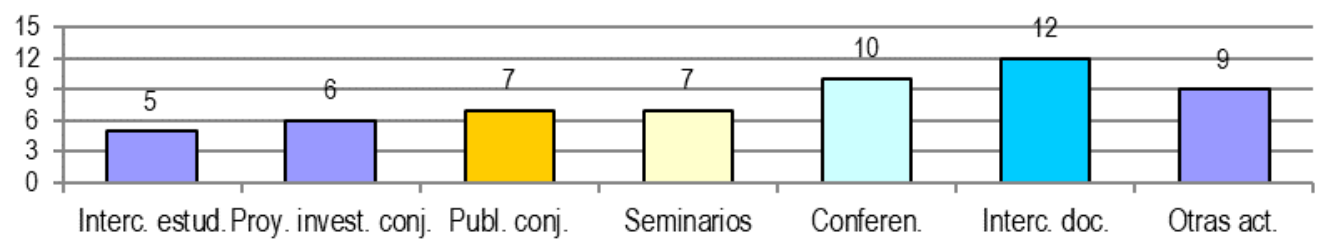

Fuente: elaboración propia con base en información obtenida de parte de las Direcciones de Posgrado informantes.

La columna titulada "Otras actividades" incluye la realización conjunta de talleres y foros, la participación en proyectos de tesis, el acceso a publicaciones y la colaboración en proyectos de investigación. Puede decirse, por consiguiente, que el espectro de actividades es bastante amplio y que incluye las acciones principales que involucran las dinámicas de internacionalización de la educación superior. 


\subsection{Docentes del programa que han realizado estudios en universidades del extranjero}

En su conjunto, el promedio general de personas que integran las plantas docentes de los 38 programas de posgrado informantes es de 20,18. De este promedio, 12,2 (60,45\%) cuentan con estudios realizados en universidades del extranjero. En términos proporcionales, es menor, en un $8 \%$, el grupo de personas con estudios realizados en el extranjero, en relación con el $68,45 \%$ de las personas directoras de los posgrados informantes que cuentan con ese tipo de estudios. No obstante, la proporción sigue siendo bastante significativa, en especial, a propósito del potencial de experiencia con el que los programas de posgrado cuentan como punto de partida para promover y llevar a cabo iniciativas y acciones de intercambio académico y de otra índole con colegas de universidades extranjeras.

\subsection{Personal docente de nacionalidad extranjera que forma parte de la planta académica del programa de posgrado}

De los 38 programas, la mitad de ellos informan tener, en su planta académica, personal docente de nacionalidad extranjera. Un programa refiere haber contado en 2015 con 9 docentes en dicha condición, mientras que otros 8 programas mencionan haber tenido una sola persona. En general, según la información obtenida, el promedio de docentes de nacionalidad extranjera en las plantas académicas de los 38 programas es de 0,63 personas por programa. En este aspecto, las diferencias entre los programas son altamente significativas, además de que, en general, la participación promedio de docentes del extranjero es también significativamente baja.

\subsection{Colegas visitantes de universidades del extranjero que participaron en actividades de los programas de posgrado informantes, en el año 2015, en docencia, investigación, acción social, conferencias, seminarios, congresos y otros}

En este aspecto, el $68,42 \%$ de los 38 programas informantes refieren haber contado en 2015 con la participación de colegas visitantes de universidades del extranjero. La cifra total es de 97 personas, para un promedio general por programa de 2,55. Según informa Carranza (2015), en ese año, en la UCR, se contó con un registro total de 193 personas académicas visitantes de universidades extranjeras (p. 11). De conformidad con esta 
información, los programas de posgrado habrían recibido, en su conjunto, la mitad del total de académicas y académicos visitantes en la UCR durante el año 2015. Estas cifras dan consistencia a la información referida en los dos anteriores acápites. Y confirman que, en efecto, tal como se señaló en el apartado de "Referencialidad teórica", es en el nivel del posgrado donde se desarrollan con mayor intensidad los intercambios académicos a escala internacional, una característica común de este nivel en las universidades latinoamericanas.

\subsection{Estudiantes del extranjero con matrícula en los programas de posgrado en 2015}

El 55,26\% de los 38 programas mencionan haber contado en 2015 con matrícula de estudiantes del extranjero. En el conjunto de los 38 programas informantes, el total de la matrícula extranjera es de 91, para un promedio general de 2,39 estudiantes por programa. Según datos que reporta la OAICE, para 2015, la cifra total de matrícula extranjera en la UCR fue de 224 estudiantes (Carranza, 2015, p. 11). De conformidad con estas cifras, la relación comparativa indica que los programas de posgrado informantes habrían contado en 2015 con el 40,62\% del total de la matrícula de estudiantes del extranjero en la UCR. De manera común y tradicional, las universidades públicas latinoamericanas, salvo pocas excepciones, presentan cifras bajas en la matrícula de estudiantes del extranjero.

\subsection{Estudiantes del programa que realizaron pasantías y asistieron a congresos y otras actividades académicas en universidades del extranjero en 2015}

23 de las 38 direcciones de posgrado informantes mencionan haber contado en 2015 con movilidad de estudiantes hacia el extranjero. Una proporción de $39,47 \%$ de los programas no reporta haber contado con ese tipo de movilidad. En total, se trata de 93 estudiantes que participaron en estas acciones de movilidad. Esta cifra representa un promedio general de 2,44 estudiantes por programa. La OAICE, por su parte, reporta que para 2015, en la UCR se registró una movilidad total de 107 estudiantes con pasantías cortas en el exterior (Carranza, 2015, p. 11). Según esto, la participación de los programas de posgrado en dicha movilidad representa el $86,91 \%$ del total registrado en la Universidad. 


\subsection{Docentes del programa que asistieron a congresos y otras actividades académicas en universidades del extranjero en 2015}

Una alta proporción de $87 \%$ de los programas informantes refieren haber contado en 2015 con movilidad docente hacia el extranjero para asistir a congresos y otras actividades académicas. En el conjunto de los 38 programas, la cifra total de docentes que participaron en acciones de movilidad hacia el exterior fue de 196, lo que representa un promedio de 5,15 docentes por programa. Esta cifra promedio refleja una alta disparidad por más del doble de académicas y académicos de los programas de posgrado que asistieron a congresos y otras actividades académicas en el extranjero, por sobre la cantidad de académicas y académicos extranjeros visitantes en 2015.

\subsection{Docentes del programa que participaron en calidad de visitantes en universidades del extranjero, en 2015, realizando actividades de docencia o participando en proyectos de investigación}

En este aspecto, las cifras representan menos de la mitad de las personas que asistieron a congresos y otras actividades en el extranjero. De los 38 programas de posgrado, el 63,15 de ellos mencionan haber contado en 2015 con movilidad de docentes hacia el extranjero para participar en actividades de docencia o en proyectos de investigación. En el conjunto de los 38 programas, dicha movilidad alcanzó, en 2015, una cifra total de 78, para un promedio de 2,05 docentes por programa. Es importante resaltar que hay una proporción significativa de programas (36,85\%) que no contaron con acciones de este tipo de movilidad en 2015. Este es un rubro de la movilidad académica que amerita ser analizado a profundidad en la UCR. La participación, sobre todo, en acciones de docencia o en proyectos de investigación es muy propicia para la conformación de colectivos académicos que pueden incrementar y diversificar las posibilidades de realizar intercambios, aspecto central para mejorar los niveles de la formación docente y las capacidades para hacer investigación en los programas de posgrado.

\subsection{Convenios de cooperación y de intercambio académico que el programa tiene suscritos y vigentes con universidades del extranjero}

En materia de convenios de cooperación y de intercambio académico suscritos con universidades del extranjero, el $39 \%$ de los programas informantes indican contar con ellos. Dentro de esta proporción, el total de convenios registrado es de 40 , distribuidos en 37 
universidades, un organismo de cooperación internacional (DAAD) y una asociación universitaria regional centroamericana (CSUCA). Las 37 instituciones con las que se tienen los convenios están distribuidas en 12 países, según como se muestra en la Figura 5.

Figura 5

Número de convenios de cooperación establecidos por los programas de posgrado de la UCR con universidades extranjeras según país. Periodo 2015-2016.

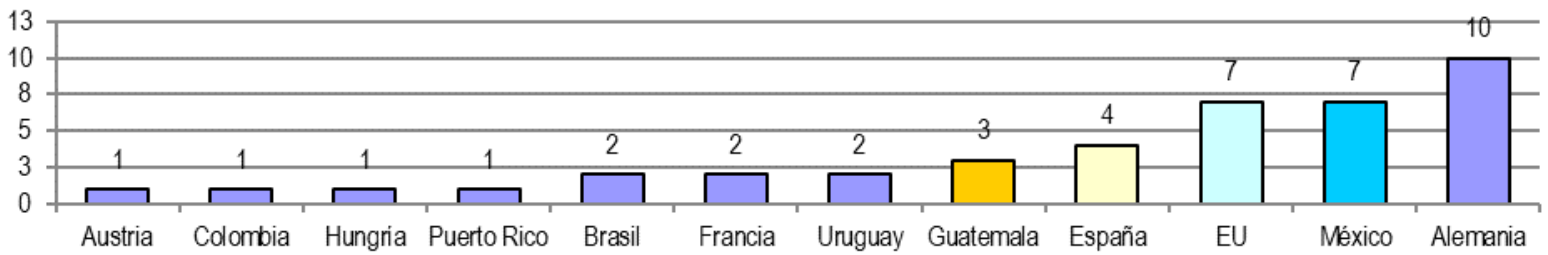

Fuente: elaboración propia con base en la información obtenida de parte de las Direcciones de Posgrado informantes.

Es de interés resaltar que 4 programas de posgrado del área de Ciencias Sociales cuentan con 16 convenios suscritos y en vigencia con universidades del extranjero: 4 convenios cada uno de esos programas. Esto representa el $40 \%$ del total de los convenios reportados por los programas informantes. Tal como es evidente, esta situación refleja una disparidad significativa en el conjunto de los programas informantes, en términos de impulsar y concretar iniciativas y acciones dirigidas a desarrollar relaciones con universidades del extranjero.

\subsection{Tipo de actividades académicas y de cooperación que se llevan a cabo en el marco de los convenios}

Dentro de las actividades académicas y de cooperación llevadas a cabo en el marco de los convenios existentes con universidades del extranjero, las que en mayor cantidad mencionan tener los programas de posgrado son las de intercambio y movilidad académica docente y estudiantil. Un total de 22 programas refieren contar con este tipo de actividades. En la Tabla 1 se indican las actividades realizadas, con el respectivo valor numérico para cada una de ellas, según la cantidad de los programas que informaron llevarlas a cabo. 
Tabla 1

Número de programas de posgrado de la UCR según actividad académica y de cooperación llevan a cabo en el marco de los convenios establecidos con universidades extranjeras. Periodo 2015-2016.

\begin{tabular}{|l|c|}
\hline Actualización curricular. & 1 \\
\hline Evaluación y autoevaluación. & 2 \\
\hline Participación en actividades de extensión o acción social. & 3 \\
\hline Proyectos conjuntos de investigación. & 5 \\
\hline Actividades de docencia como académica o académico visitante. & 6 \\
\hline Asesoría académica. & 7 \\
\hline Intercambio y movilidad académica docente. & 10 \\
\hline Intercambio y movilidad académica estudiantil. & 12 \\
\hline
\end{tabular}

Fuente: Elaboración propia con base en la información obtenida de parte de las Direcciones de Posgrado informantes.

\subsection{Beneficios que aportan al programa de posgrado los convenios de cooperación y de intercambio académico establecidos con universidades del extranjero}

En la Tabla 2 se presenta un listado de los beneficios que, según indican los programas informantes, proporcionan los convenios suscritos con universidades del extranjero. En la columna de la derecha se incluye el valor numérico relativo a la cantidad de los programas que en cada caso emitieron su valoración.

Tabla 2

Número de programas de posgrado de la UCR según beneficio que propicia los convenios establecidos con universidades extranjeras. Periodo 2015-2016.

\begin{tabular}{|l|l|}
\hline Fortalecen las capacidades para la gestión administrativa del programa & 3 \\
\hline Ayudan a la mejora de las capacidades de gestión académica del programa & 7 \\
\hline Diversifican las líneas temáticas de investigación & 7 \\
\hline Favorecen la colocación contextualizada en el plan de estudios de los contenidos de formación & 7 \\
\hline Contribuyen al desarrollo de la sensibilidad intercultural del estudiantado & 8 \\
\hline Favorecen la participación en redes académicas internacionales & 9 \\
\hline Mejoran la pertinencia del programa & 9 \\
\hline Fortalecen la investigación & 10 \\
\hline Contribuyen a diversificar la proyección internacional del programa & 11 \\
\hline Enriquecen la docencia con nuevos enfoques y prácticas pedagógicas & 12 \\
\hline Mejoran la calidad del quehacer académico del programa & 13 \\
\hline
\end{tabular}

Fuente: Elaboración propia con base en información obtenida de parte de las Direcciones de Posgrado informantes. 


\subsection{Participación en redes académicas internacionales}

De las 38 direcciones de posgrado informantes, 14 (36,84\%) de ellas mencionan tener participación del programa en redes académicas internacionales. Es una proporción que se puede estimar como baja, teniendo en cuenta la experiencia en relaciones internacionales con que cuentan quienes ocupan las direcciones de los programas, así como, en general, quienes forman parte de las correspondientes plantas académicas de cada uno de ellos. Las temáticas de las redes son diversas, según se muestra en la Tabla 3.

Tabla 3

Número de programas de posgrado de la UCR según las redes temáticas en las que participan.

Periodo 2015-2016.

\begin{tabular}{|l|c|}
\hline \multicolumn{1}{|c|}{ Nombre de la red temática } & $\begin{array}{c}\text { Cantidad } \\
\text { programas } \\
\text { participantes }\end{array}$ \\
\hline Red de Monitoreo y Evaluación de América Latina y el Caribe (REDLACME) & 1 \\
\hline Políticas de Educación Superior & 1 \\
\hline Red Interamericana de Educación en Administración Pública (INPAE) & 1 \\
\hline Red Académica Internacional en Desarrollo de la Antropología en Centroamérica & 1 \\
\hline Sociedad Europea de Evaluación & 1 \\
\hline $\begin{array}{l}\text { Red de Seguimiento, Evaluación y Sistematización de América Latina y el Caribe } \\
\text { (RELAC). }\end{array}$ & 1 \\
\hline Extensión o Acción Social & 2 \\
\hline Políticas y estrategias de gestión de los estudios de posgrado & 2 \\
\hline Homologación de planes de estudio & 2 \\
\hline Evaluación y acreditación de programas de posgrado & 2 \\
\hline Docencia & 4 \\
\hline Investigación & 7 \\
\hline
\end{tabular}

Fuente: Elaboración propia con base en información obtenida de parte de las Direcciones de Posgrado informantes.

\subsection{Valoración acerca de la participación en redes académicas internacionales}

De manera puntual, 21 (55,26\%) direcciones de posgrado aportaron su valoración acerca de la participación del programa en redes académicas internacionales. En la Tabla 4 se muestran los resultados con el correspondiente valor numérico según el respectivo ítem de valoración. 
Tabla 4

Número de programas de posgrados según valoración de la participación en redes académicas internacionales. Periodo 2015-2016.

\begin{tabular}{|l|c|}
\hline $\begin{array}{l}\text { Poco funcionales por generalización de procedimientos que no siempre se adaptan a todas } \\
\text { las universidades ni países }\end{array}$ & 1 \\
\hline Limitan la independencia y autonomía académica del programa de posgrado & 2 \\
\hline $\begin{array}{l}\text { Permite la identificación de fuentes alternativas de financiación para fortalecer la movilidad } \\
\text { académica docente y estudiantil }\end{array}$ & 5 \\
\hline $\begin{array}{l}\text { Establecen dinámicas que tienden a afectar las directrices académicas (plan de estudios, } \\
\text { investigación) definidas por el programa de posgrado }\end{array}$ & 6 \\
\hline Contribuye a enriquecer la docencia & 8 \\
\hline Favorece la realización de proyectos conjuntos de investigación & 10 \\
\hline $\begin{array}{l}\text { Amplía las posibilidades para establecer relaciones con programas homólogos en } \\
\text { universidades del extranjero }\end{array}$ & 10 \\
\hline Facilita alternativas de movilidad docente y estudiantil & 12 \\
\hline Diversifica las alternativas de intercambio académico & 12 \\
\hline Mejora la proyección internacional del programa de posgrado & 12 \\
\hline Favorece el fortalecimiento académico del programa de posgrado & 15 \\
\hline
\end{tabular}

Fuente: Elaboración propia con base en información obtenida de parte de las Direcciones de Posgrado informantes.

\subsection{Acerca de la internacionalización en los programas de posgrado: Beneficios, perjuicios, dificultades y otras consideraciones complementarias}

\subsubsection{Beneficios}

A este ítem de pregunta, proporcionaron respuesta 29 direcciones de posgrado. El ítem estuvo constituido por 9 aspectos, de los cuales 5 de ellos obtuvieron respuesta afirmativa por parte de 20 o más direcciones. En la Tabla 5, se indican los aspectos considerados, con el respectivo puntaje obtenido según la cantidad de programas que los refieren. 
Tabla 5

Número de programas de posgrados de la UCR que indican los beneficios que le aporta la internacionalización. Periodo 2015-2016.

\begin{tabular}{|l|c|}
\hline Favorece la mejora de capacidades para atraer personal académico del extranjero & 12 \\
\hline Fortalece la cultura e identidad académica institucional del programa & 15 \\
\hline Mejora la cooperación y la solidaridad interinstitucional a escala internacional & 17 \\
\hline Contribuye a mejorar la gestión académica & 17 \\
\hline Aumenta la orientación internacional del personal académico & 20 \\
\hline $\begin{array}{l}\text { Contribuye a diversificar los enfoques de la investigación y a incorporar la } \\
\text { interdiscinlinariedad }\end{array}$ & 21 \\
\hline $\begin{array}{l}\text { Favorece la realización de publicaciones conjuntas con colegas de universidades del } \\
\text { extraniero }\end{array}$ & 21 \\
\hline Incrementa la conciencia internacional de la planta docente y del estudiantado & 21 \\
\hline Fortalece la proyección internacional del programa de posgrado & 22 \\
\hline
\end{tabular}

Fuente: Elaboración propia con base en información obtenida de parte de las Direcciones de Posgrado informantes.

\subsubsection{Perjuicios}

Respecto de los perjuicios que la internacionalización puede provocar al programa, las respuestas a este ítem son escasas: 13 en total. Se indican como perjuicios los siguientes: a) incide en modificaciones al plan de estudios que socavan los principios académicos y la pertinencia social de la formación que brinda el programa (2 respuestas), b) incentiva la emigración de personal académico y de estudiantes ("fuga de cerebros") (2 respuestas), c) introduce agendas de investigación que condicionan al programa a plegarse al estudio de problemas que responden a intereses externos (4 respuestas), d) conduce a generar dependencias financieras respecto de instituciones y universidades del extranjero (5 respuestas).

En términos generales, en consecuencia, las Direcciones de Posgrado informantes estiman que la internacionalización genera una variedad importante de beneficios y muy escasos perjuicios.

\subsubsection{Dificultades}

Respecto de las dificultades para desarrollar acciones de internacionalización, las más importantes, mencionadas en las respuestas, son las que se indican en la Tabla 6. 
Tabla 6

Número de programas de posgrado de la UCR que afrontan dificultades para desarrollar acciones de internacionalización. Periodo 2015-2016.

\begin{tabular}{|l|l|}
\hline $\begin{array}{l}\text { Falta de recurso humano para desarrollar y dar seguimiento a las acciones de } \\
\text { internacionalización }\end{array}$ & 1 \\
\hline Falta de tiempo & 1 \\
\hline Interés limitado de parte de la población estudiantil & 6 \\
\hline Dificultades para el reconocimiento académico de la implicación internacional & 7 \\
\hline Poco interés e involucramiento de parte del personal docente & 7 \\
\hline $\begin{array}{l}\text { Plan de estudios poco flexible para participar en programas centrados en la } \\
\text { internacionalización }\end{array}$ & 9 \\
\hline Falta de estructura organizativa & 9 \\
\hline Visión institucional limitada & 13 \\
\hline Carencia de estrategia y de un plan para guiar el proceso de internacionalización & 14 \\
\hline Falta de políticas y procedimientos institucionales & 17 \\
\hline Inercia administrativa y dificultades burocráticas & 20 \\
\hline Financiación insuficiente & 23 \\
\hline
\end{tabular}

Fuente: Elaboración propia con base en información obtenida de parte de las Direcciones de Posgrado informantes.

De acuerdo con lo anterior, las principales dificultades estarían asociadas con aspectos básicos de la internacionalización, tales como: financiación, gestión y procedimientos administrativos, insuficiencia de políticas institucionales, carencia de estrategias y planes para orientar las acciones de internacionalización.

\subsubsection{Consideraciones complementarias sobre la internacionalización}

En este acápite se refieren las consideraciones que algunas de las direcciones de programas de posgrado hicieron con respecto a las condiciones con que se cuenta o se debería contar para desarrollar acciones de internacionalización. Son las siguientes y se transcriben de manera literal, salvo en los casos en que hubo necesidad de hacer correcciones de forma.

1. "El cargo de la dirección de posgrado es de 1/2 tiempo completo; el otro 1/2 tiempo lo aporta la unidad académica a la que el programa pertenece. En nuestro caso, con ese 1/2 tiempo se atienden dos modalidades de titulación: académica y profesional. Se cuenta además con únicamente 1/2 tiempo para una persona que da apoyo en calidad 
de asistente de la dirección. Esto quiere decir que a la dirección le toca hacerlo todo, pues el respaldo que se obtiene por parte del SEP es de tiempos docentes para los cursos que se imparten semestre a semestre. A menos que la unidad académica tenga dentro de su plan estratégico la internacionalización, es muy difícil que un posgrado la incorpore".

2. “El posgrado en la regionalización universitaria nos obliga a desarrollar otras estrategias. Para lograr objetivos de internacionalización, por iniciativa propia, nosotros tenemos tanto estudiantes como pasantes de otros países. Se cuenta para ello con una ayuda mínima de parte de las correspondientes instancias de la Universidad. En nuestro caso, nos visitan docentes del extranjero y hemos salido del país utilizando otras fuentes de financiamiento. Esto no quiere decir que no utilicemos lo que está institucionalmente establecido. No obstante, en las Sedes Regionales aplicamos otras formas de gestión que nos permiten ahorrar tiempo".

3. “EI SEP debería organizar y gestionar un programa de internacionalización del posgrado de la UCR en sus distintos componentes: estructura organizativa, financiamiento, movilidad académica, convenios, cooperación internacional, posgrados compartidos."

4. "Existe un gran interés en mi gestión por proyectar y brindar una oferta donde el posgrado tenga una proyección internacional. Mi tropiezo ha sido que el Posgrado es de financiamiento complementario y, además, presencial. Esto repercute en que no represente una buena opción para estudiantes extranjeros, además de que para docentes de universidades de otros países no es viable la participación por existir un salario insuficiente".

5. "La internacionalización es importante, pero muchas veces nos vemos amarrados por la tramitología. Esto desmotiva mucho a la planta académico".

6. “La internacionalización puede ser buena. No obstante, también debe motivarse al personal docente a cargo para que participe en docencia e investigación de posgrado en nuestra propia realidad nacional. Si nuestros propios programas no están fortalecidos en relación con lo que la realidad local y nacional exige, ¿cómo podríamos articularnos con otros programas en universidades del extranjero? Me parece que mucho se busca proyectarse hacia afuera, entretanto que se tiende a menospreciar lo interno, y no siempre lo externo es mejor y de mayor calidad".

7. "La OAICE urge de una jefatura propicia en la promoción y el intercambio y menos imposición, burocracia y preeminencia de los encargados asistentes de áreas". 
8. "Me parece que debe ser mandatorio que las matriculas reserven cupos para estudiantes extranjeros, a fin de que estos no lleguen a competir por una misma matrícula con los estudiantes nacionales".

9. "Puede convertirse en un valioso recurso, pero, como complemento, no como centro de acciones, dado que es un proceso cuya continuidad es muy sensible a la falta de presupuesto o a la normativa regional".

10. "Uno de los retos es el poco apoyo con que contamos en el Posgrado, donde los informes de la Oficina de Administración Financiera (OAF) sobre los recursos no son adecuados; donde hay que luchar por los espacios físicos y ver que los estudiantes que pagan 50.000 colones por crédito no tienen donde estacionarse; donde los profesores tienen graves problemas de pago y donde cualquier iniciativa de mejora choca con la realidad institucional; donde los recursos que generamos no los podemos usar para fortalecer el programa, ni siquiera para pagar un curriculista. Entonces, cuando se plantea traer un profesor invitado o atraer estudiantes extranjeros (tenemos gente de Angola, México), está uno tentado a no tomar iniciativa".

\section{Consideraciones finales}

1. Según se pudo ver al inicio del apartado de "Resultados", en los 38 programas de posgrado informantes, existe una proporción de 68\% de personas que ocupan cargos de dirección, quienes han realizado los estudios de su última titulación en una universidad del extranjero. También, es importante subrayar que únicamente una de estas personas realizó sus estudios en una universidad de América Latina.

De este grupo de personas, que realizaron estudios de posgrado en universidades del extranjero, $80 \%$ de ellas mencionan que, en diversos grados y modalidades, mantienen relaciones de índole académica con colegas de dichas universidades. Las relaciones establecidas están siendo aprovechadas para incorporarlas a la dinámica académica de los programas de posgrado mediante diversas actividades de intercambio y de labor académica conjunta: a) intercambio docente, b) conferencias; c) seminarios; d) publicaciones conjuntas; e) proyectos de investigación conjuntos; f) intercambio estudiantil.

A propósito del desarrollo de capacidades y estrategias de internacionalización en los programas de posgrado, puede afirmarse que la anterior proporción representa una base positiva para ser aprovechada, con la finalidad de fortalecer las acciones y las relaciones de 
intercambio y colaboración académica internacional en las distintas áreas de la docencia, la investigación, la acción social, la gestión académica y otras.

Dicha experiencia de estudios en el extranjero, también es importante subrayarlo, se ha desarrollado inscrita en las rutas tradicionales que en las universidades de América Latina son comunes, cuando se trata de elegir universidades y países de destino para realizar estudios de posgrado en el extranjero: son predominantemente las universidades de los países del hemisferio de América del Norte y Europa. Esta tradición se ve reforzada, actualmente, en la política institucional de la UCR, cuando, a propósito de la suscripción de nuevos convenios con universidades del extranjero, en el Plan Estratégico Institucional 2013/2017, se hace énfasis en establecer relaciones con "macrouniversidades de prestigio internacional“.

La diversificación de las rutas de destino para realizar estudios en universidades del extranjero (maestrías, doctorados, postdoctorados), en la UCR, en consecuencia, se ve restringida en la política institucional y en las acciones que involucran los programas a cargo de la Oficina de Asuntos Internacionales y Cooperación Externa (OAICE), instancia de la Rectoría a cargo de tramitar y gestionar la suscripción de convenios, los programas de becas y la movilidad docente, estudiantil y administrativa.

Es importante que la universidad, por medio de las diferentes instancias institucionales a las que corresponda, mantenga y busque mejorar, no solo la movilidad docente hacia el exterior y el intercambio académico con universidades del extranjero, sino que, además, se proporcione asesoría y apoyo equitativo y desburocratizado a todos los distintos programas de posgrado para que puedan mejorar las capacidades y las estrategias requeridas para el establecimiento de relaciones internacionales de intercambio y colaboración con universidades, unidades académicas y grupos de investigación de universidades del extranjero.

Asimismo, la dimensión de la movilidad estudiantil es otro aspecto muy importante para los programas de posgrado. La información obtenida en la presente ocasión dibuja una situación que podría calificarse como requerida de mayor apoyo y mejora. Tan solo el 60\% de los programas informantes refieren haber contado en 2015 con movilidad de estudiantes hacia el exterior, para ir a realizar pasantías o asistir a congresos y otras actividades académicas.

Esta situación puede decirse que representa un desafío para la gestión académica e institucional, tanto en lo que concierne a la gestión que llevan a cabo las direcciones de los 
programas de posgrado como en lo que corresponde a las funciones que tienen a su cargo la Decanatura del SEP y la OAICE.

2. Una cuestión importante, relativa a las capacidades y estrategias de internacionalización a desarrollar por parte de los programas de posgrado, la representa el contar con la disposición de convenios de cooperación suscritos con universidades del extranjero. En el caso de la presente investigación, únicamente el $38,47 \%$ de las 38 direcciones de posgrado informantes indicaron tener este tipo de relaciones establecidas. Según se refirió ya en el punto "IV.11“, el $80 \%$ de esos convenios están suscritos con universidades de Europa y América del Norte, y se encuentran concentrados en un pequeño grupo de 12 países.

Esta situación es concomitante, con la ya señalada, a propósito de la movilidad académica para realizar estudios en el extranjero. Se confirma la existencia institucional de la UCR en un imaginario en el que las universidades del Norte son las que aparecen ostentando los lugares de privilegio. Los estudios en el extranjero no solo involucran la obtención de una titulación o la exclusiva experiencia de la formación académica, también implican otro tipo de experiencias, las cuales contribuyen al enriquecimiento integral de la formación en las personas: en lo cultural, la visión de mundo, la perspectiva de vida y la identidad de cada cual. En este sentido, puede decirse que recalcar el referido imaginario de que es en las universidades del Norte y en las "macrouniversidades de prestigio" donde deben enfocarse las preferencias soslaya de manera absoluta esta segunda dimensión, que es igual o, incluso, aún más importante que la dimensión estrictamente académica.

En el caso de los programas de posgrado, es importante y pertinente, con mayor razón, desarrollar mayores relaciones de cooperación y de intercambio colaborativo, tal como propone la CRES (2008), en la escala regional. Por una parte, hay una repercusión positiva de financiación: los costos de la movilidad y el intercambio académico disminuyen de manera significativa. Esto permitiría facilitar condiciones de acceso a la movilidad para una mayor cantidad de personas y con los mismos montos de presupuesto. Por otra parte, en el contexto de América Latina se comparte, en gran medida, una matriz común de problemas y de situaciones que vienen a ser relevantes para el desarrollo de los procesos de formación, la investigación y la acción social en los programas de posgrado.

En los aspectos propiamente de gestión, también es necesaria una promoción y facilitación mayor de oportunidades para que los programas de posgrado cuenten con 
convenios de cooperación y de intercambio colaborativo con universidades del extranjero. El intercambio y la colaboración académica, en el marco de los convenios, contribuyen a favorecer condiciones para la mejora de la excelencia académica, allegan elementos para enriquecer la docencia con nuevos enfoques y prácticas pedagógicas, amplían perspectivas para realizar una colocación contextualizada de los contenidos del plan de estudios, aportan ingredientes para fortalecer la investigación y la pertinencia social del quehacer académico de los programas.

3. Tal como ya se mencionó en el apartado de "Referencialidad teórica“, una figura que ha tomado una marcada importancia durante los últimos años es la utilización de redes académicas internacionales. Desde hace al menos tres décadas, las redes empezaron a ser promovidas y adoptadas como un instrumento propicio para desarrollar y articular intercambios académicos cooperativos y colaborativos entre las universidades a escala internacional. Hay quienes las ven con alguna reticencia, en la medida que se les atribuye estar constituidas para fines con carácter muy específico. Además de que tienden a ser en exceso inestables y de breve temporalidad. Otro rasgo que se les atribuye es el de contar con una alta movilidad de la membresía, ya que las personas están llegando y otras se están yendo con marcada frecuencia. Con todo, en la medida en que las redes sean aprovechas como punto de partida para establecer nuevas relaciones y diversificar las ya existentes, la participación en ellas puede llegar a ser de beneficio para los programas de posgrado, las unidades académicas y la universidad en su conjunto.

En los resultados de investigación aquí referidos, la participación en redes académicas internacionales presenta una proporción todavía baja: 36,84\% del total de los programas de posgrado informantes indican tenerla. Esta relación puede calificarse como débil, considerando que se trata del nivel del posgrado, una dimensión del quehacer académico en la universidad que amerita, en mayor medida, mantener una dinámica diversa de relaciones internacionales en docencia, investigación, acción social y gestión académica. Al respecto, es importante destacar que la valoración realizada acerca de las redes por parte de los programas informantes es altamente positiva, se menciona, además, una apreciable variedad de temáticas en las que se participa.

Las redes académicas internacionales favorecen la diversificación de las alternativas de intercambio académico (docente y estudiantil), amplía las posibilidades para establecer relaciones con programas homólogos en universidades del extranjero, así como contribuyen 
a enriquecer la docencia y la investigación por medio del desarrollo de acciones conjuntas. Pueden ser consideradas como un importante punto de partida para desarrollar acciones de internacionalización que, de manera planificada, permitan ir consolidando relaciones con colegas del extranjero y que, aunado a ello contribuyan a un mayor desarrollo de los intercambios.

Ahora bien, teniendo en cuenta la baja participación que los programas de posgrado de la UCR informan tener en redes académicas internacionales, parece pertinente sugerir la necesidad de hacer una revisión en detalle respecto de las posibilidades de financiamiento con las que se cuenta, la gestión académica y administrativa que se está llevando a cabo, la definición de políticas institucionales y de programas y procesos sistémicos articulados, la existencia de un plan de internacionalización que sea acorde con las características institucionales, la cultura académica y los principios misionales y estatutarios que rigen el desempeño y la razón de ser de la Universidad. Es necesaria, además, la facilitación institucional de oportunidades para que, de una manera equitativa, los diferentes programas de posgrado puedan participar en proyectos y actividades en red.

\section{Agradecimientos}

A la Dra. Cecilia Díaz Oreiro, quien, como Decana del SEP, le brindó un valioso respaldo a la realización de este proyecto.

A la Master Diana Arce Flores, funcionaria del SEP que colaboró con este proyecto haciendo la revisión y validación del instrumento de investigación. Sus observaciones y recomendaciones fueron muy valiosos para hacerle ajustes y mejoras.

Al Master Franklin Rodríguez Vargas, funcionario del Instituto de Investigación en Educación (INIE) de la UCR, quien colaboró con el proyecto para la colocación en línea del instrumento de investigación.

Al equipo de investigación del Observatorio de la Educación Nacional y Regional (OBSED) del INIE, que con sus comentarios y observaciones contribuyó en gran medida a orientar la elaboración de la propuesta del proyecto y a la mejora del presente artículo. 


\section{Referencias}

Albizu Ontaneda, Alejandra. (2014). La internacionalización de la educación superior. Un recorrido conceptual. En: Tangelson, Guillermo (comp.). (2014). Desde el Sur: miradas sobre la internacionalización (pp. 83-103). Buenos Aires: Ediciones de la UNLa. Recuperado de http://www.cinda.cl/wp-content/uploads/2014/09/Desde-el-sur-miradassobre-la-internacionalizaci\%C3\%B3n.pdf

Altbach, Philip G. y Knight, Jane. (2006). Visión panorámica de la internacionalización en la educación superior: motivaciones y realidades. Perfiles Educativos Mexicanos, 28(112), 13-39. Recuperado de http://www.scielo.org.mx/pdf/peredu/v28n112/n112a2.pdf

Carranza Velázquez, Julieta. (2015). Informe de labores 2015. San José: OAICE/Universidad de Costa Rica. Recuperado de: http://www.oaice.ucr.ac.cr/archivos/OAICEINFORME DE LABORES-2015.pdf

Consejo Universitario de la Universidad de Costa Rica. (2005). Estatuto Orgánico de la Universidad de Costa Rica. Recuperado de: http://www.cu.ucr.ac.cr/estatutoorganico.html

Conferencia Regional de la Educación Superior (CRES). (2008). Declaración Final de la Conferencia Regional de Educación Superior en América Latina y el Caribe. Caracas: IESALC/UNESCO. Recuperado de http://www.unesco.org.ve/index.php?option=com content\&view=article\&id=365\&ltemid $=423 \&$ lang $=$ es

Dias, Marco Antonio R. (2008). La internacionalización y la cooperación universitaria en la sociedad del conocimiento. En Carlos Tünnermann Bernheim (ed.), La educación superior en América Latina y el Caribe: diez años después de la Conferencia Mundial de 1998 (pp. 313-366). Cali: Sello Editorial Javeriano/IESALC.

Didou, Sylvie. (2005). Internacionalización y proveedores externos de educación superior en los países de América Latina y en el Caribe: principales problemáticas. Ciudad de México: Departamento de Investigaciones Educativas del CINVESTAV. Recuperado de https://eco.mdp.edu.ar/cendocu/repositorio/00119.pdf

Escrigas, Cristina y Lobera, Josep. (2009). Introducción. Nuevas dinámicas para la responsabilidad social. En Global University Network for Innovation. (2009). La educación superior en tiempos de cambio. Nuevas dinámicas para la responsabilidad social (pp. 3-16). Madrid: Mundi-Prensa. Recuperado de http://upcommons.upc.edu/bitstream/handle/2099/9540/ESM Sin 09.pdf?sequence=6

Lanz, Rigoberto, Fergusson, Alex y Marcuzzi, Arianna. (2006). Procesos de reforma de la educación superior en América Latina. En: Instituto Internacional para la Educación Superior en América Latina y el Caribe. (2006). Informe sobre la educación superior en América Latina y el Caribe. 2000-2005 (pp. 105-114). Caracas: Editorial Metrópolis. 
Mollis, Marcela. (2010a). Imágenes de posgrado: entre la academia, el mercado y la integración regional. En Marcela Mollis, Jorge Núñez Jover y Carmen García Guadilla. (2010). Políticas de posgrado y conocimiento público en América Latina y el Caribe: desafíos y perspectivas (pp. 13-56). Buenos Aires: CLACSO e Instituto de Investigaciones Gino Germani.

Mollis, Marcela. (2010b). Las transformaciones de la educación superior en América Latina: ¿identidades en construcción? Educación Superior y Sociedad, 15(1), 11-22. Recuperado de http://unesdoc.unesco.org/images/0019/001917/191731m.pdf

Nussbaum, Martha C. (2012). Crear capacidades. Propuesta para el desarrollo humano. Barcelona: Paidós.

Oficina de Planificación Universitaria, Universidad de Costa Rica. (s.f.). Plan Estratégico Institucional 2013/2017. Recuperado de: Recuperado de: http://www.oplau.ucr.ac.cr/phocadownload/plan estrategico/Plan estrategico institucio nal 2013-2017.pdf

Vessuri, Hebe. (2003). La ciencia y la educación superior en el proceso de internacionalización. Elementos de un marco conceptual para América Latina. París: Foro de la UNESCO. Recuperado de http://unesdoc.unesco.org/images/0013/001347/134783so.pdf

Vessuri, Hebe. (2008). Competición y colaboración en contexto de multiplicación de "centros de atracción" y "desiertos yermos". Revista de la Educación Superior, vol. 37(148), 123139. Recuperado de: http://www.redalyc.org/articulo.oa?id=60416038009 